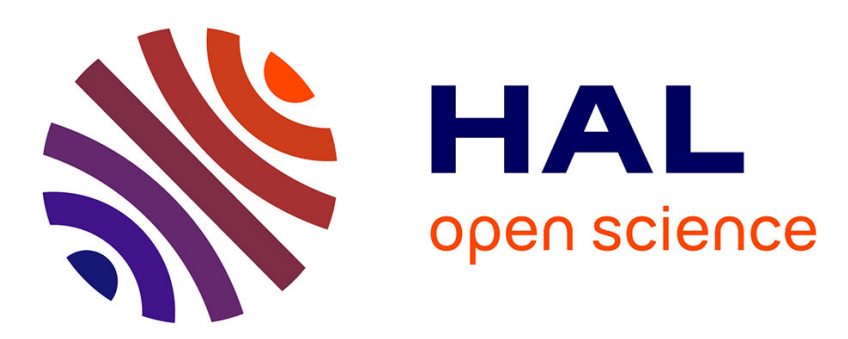

\title{
Evidence of slippage breakdown for a superhydrophobic microchannel
}

Guido Bolognesi, Cécile Cottin-Bizonne, Christophe Pirat

\section{To cite this version:}

Guido Bolognesi, Cécile Cottin-Bizonne, Christophe Pirat. Evidence of slippage breakdown for a superhydrophobic microchannel. Physics of Fluids, 2014, 26 (8), pp.082004. 10.1063/1.4892082 . hal-02313467

\section{HAL Id: hal-02313467 \\ https://univ-lyon1.hal.science/hal-02313467}

Submitted on 4 Feb 2021

HAL is a multi-disciplinary open access archive for the deposit and dissemination of scientific research documents, whether they are published or not. The documents may come from teaching and research institutions in France or abroad, or from public or private research centers.
L'archive ouverte pluridisciplinaire HAL, est destinée au dépôt et à la diffusion de documents scientifiques de niveau recherche, publiés ou non, émanant des établissements d'enseignement et de recherche français ou étrangers, des laboratoires publics ou privés. 


\section{AIP | Physics of Fluids}

\section{Evidence of slippage breakdown for a superhydrophobic microchannel}

G. Bolognesi, C. Cottin-Bizonne, and C. Pirat

Citation: Physics of Fluids (1994-present) 26, 082004 (2014); doi: 10.1063/1.4892082

View online: http://dx.doi.org/10.1063/1.4892082

View Table of Contents: http://scitation.aip.org/content/aip/journal/pof2/26/8?ver=pdfcov

Published by the AIP Publishing

\section{Articles you may be interested in}

Effective slippage on superhydrophobic trapezoidal grooves

J. Chem. Phys. 139, 174708 (2013); 10.1063/1.4827867

Eccentricity effects of microhole arrays on drag reduction efficiency of microchannels with a hydrophobic wall Phys. Fluids 24, 112004 (2012); 10.1063/1.4767539

Pressure and partial wetting effects on superhydrophobic friction reduction in microchannel flow Phys. Fluids 24, 112003 (2012); 10.1063/1.4767469

Frictional slip lengths for unidirectional superhydrophobic grooved surfaces

Phys. Fluids 23, 072001 (2011); 10.1063/1.3605575

On the effects of liquid-gas interfacial shear on slip flow through a parallel-plate channel with superhydrophobic grooved walls

Phys. Fluids 22, 102002 (2010); 10.1063/1.3493641

\section{A|P| $\left.\right|_{\text {Applied Physics }} ^{\text {Journal of }}$}

Journal of Applied Physics is pleased to announce André Anders as its new Editor-in-Chief 


\title{
Evidence of slippage breakdown for a superhydrophobic microchannel
}

\author{
G. Bolognesi, ${ }^{\text {a) }}$ C. Cottin-Bizonne, and C. Pirat \\ ILM, Université de Lyon, Université de Lyon 1 and CNRS, UMR5306, \\ F-69622 Villeurbanne, France
}

(Received 5 October 2013; accepted 17 July 2014; published online 13 August 2014)

\begin{abstract}
A full characterization of the water flow past a silicon superhydrophobic surface with longitudinal micro-grooves enclosed in a microfluidic device is presented. Fluorescence microscopy images of the flow seeded with fluorescent passive tracers were digitally processed to measure both the velocity field and the position and shape of the liquid-air interfaces at the superhydrophobic surface. The simultaneous access to the meniscus and velocity profiles allows us to put under a strict test the no-shear boundary condition at the liquid-air interface. Surprisingly, our measurements show that air pockets in the surface cavities can sustain non-zero interfacial shear stresses, thereby hampering the friction reduction capabilities of the surface. The effects of the meniscus position and shape as well as of the liquid-air interfacial friction on the surface performances are separately assessed and quantified. (C) 2014 AIP Publishing LLC. [http://dx.doi.org/10.1063/1.4892082]
\end{abstract}

\section{INTRODUCTION}

In lab-on-a-chip technology, the high hydrodynamic resistance the liquid experiences as it flows in a network of micrometric channels often imposes the microfluidic device to be connected to pumping systems thousands of times bigger than the chip itself, thereby erasing many advantages of a millimeter-sized device. In this respect, superhydrophobic surfaces (SHS) have demonstrated to be an effective tool to relax the constraint of no-moving liquid at the channel walls, ${ }^{1}$ due to air pockets trapped in surface cavities (the so-called Cassie or Fakir state). Air bubbles durably reside in the surface roughness only under specific conditions of pressure and surface wettability. ${ }^{2}$ When the Cassie state is no longer stable, the meniscus collapses within the cavities ${ }^{3-5}$ and the liquid is in contact with the entire exposed surface of the solid (the so-called Wenzel state).

In literature, the experimental measurements of water slippage past SHS, usually characterized in terms of effective slip length, ${ }^{6}$ spread over a quite broad data range which spans from hundreds of nanometers ${ }^{7}$ to few hundreds of micrometers. ${ }^{8}$ Slight discrepancies between measurements and theoretical predictions are also reported and mainly ascribed to experimental factors which cannot always be easily controlled or quantified. One of those is certainly the meniscus deformation ${ }^{9,10}$ resulting from the balance of liquid pressure and surface tension. Theoretical ${ }^{11-15}$ studies have demonstrated the negative impact of the meniscus curvature and position on the effective slip length. Such a detrimental effect has been confirmed by experimental investigations of SHS characterized via several techniques like confocal microscopy, ${ }^{16}$ micro particle image velocimetry $(\mu$-PIV $),{ }^{17}$ pressure versus flow rate measurements, ${ }^{18}$ and dynamic surface force apparatus. ${ }^{19}$ Similar results were also reported by numerical analysis via continuum models, ${ }^{20}$ molecular dynamics, ${ }^{21}$ and Lattice-Boltzmann simulations. ${ }^{22}$ Very recently, a superhydrophobic microfluidic device with active control of the meniscus shape has been presented. ${ }^{23}$ Through numerical and experimental

\footnotetext{
a)g.bolognesi@imperial.ac.uk. Present address: Department of Chemistry, Imperial College London, South Kensington Campus, SW7 2AZ, London, United Kingdom.
} 
investigations, the authors showed how the resulting effective slip length could be tuned and optimized by controlling the protrusion depth of the meniscus into the flow.

Another phenomenon which might be responsible for a dramatic increase in the apparent surface friction, but has not yet received adequate attention in the study of SHS, is the fact that a waterair interface cannot always be treated as a stress free boundary. In that respect, there are quite few experimental evidences ${ }^{18,24-28}$ showing that at the micrometer scale partial-slip or even no-slip assumptions can be more appropriate, in some circumstances, for modelling water-air interface under experimental conditions. Lazouskaya et al. ${ }^{24}$ were the first to report a reduced mobility at the liquidair interface in an open capillary channel, questioning the validity of the no-shear boundary condition at the meniscus interface. More recently, the same research group performed new experiments with a very similar geometry ${ }^{29}$ and highlighted the dependence of an effective interfacial shear stress on the channel dimensions. Particularly, for channel width below the water capillary length (i.e., $2.7 \mathrm{~mm}$ ), the interfacial shear stress increases as the channel size decreases. Investigating the speed of the advancing meniscus flooding an open hydrophilic channel, Yang et al. ${ }^{30}$ found a good agreement between experimental data and theoretical predictions when a no-slip boundary condition was assumed at the liquid-air interface.

Since the benefits of SHS in terms of friction reduction rely on the presence of stress-free boundaries, it is essential to probe whether or not the liquid-air interfaces can always be considered as a perfect slip interface. In this paper, we fully characterize the flow past a silicon micro-grooved surface and we specifically focus our attention on the interfacial friction behaviour of the liquidair interfaces. However, discriminating between the effects of a deformed meniscus, on one side, and the interfacial friction at the liquid-air interface, on the other side, is not an easy task as this requires measuring the flow nearby the meniscus while simultaneously determining its shape and position. Experimental configurations to probe both the liquid flow and the meniscus deformation have been recently introduced. ${ }^{18,23,31}$ For all those studies, the direction normal to the SHS has to be co-planar to the microscope focal plane. This necessarily limits the experimental investigation to one single surface geometry, that is the one with micro-grooves perpendicular to the flow direction. Micro-structured surfaces with micro-grooves parallel to the flow direction or with pillars and holes would be much more difficult to be studied in that experimental configuration. In this respect, we recently developed a novel technique ${ }^{32}$ to perform simultaneous velocity and interface profile measurements on SHS. That technique has no restriction on the SHS orientation and it has been successfully used to study even very complicated surface pattern like ellipse-shaped pillars. We now adopt such a technique to characterize the interfacial friction of a water flow past a silicon surface with longitudinal micro-grooves (see Fig. 1). We first investigate the slippage of the SHS globally by measuring the effective slip length of the velocity profile averaged over a surface pitch. We then focus on the local interfacial friction and we evaluate the friction contributions of the liquid-solid

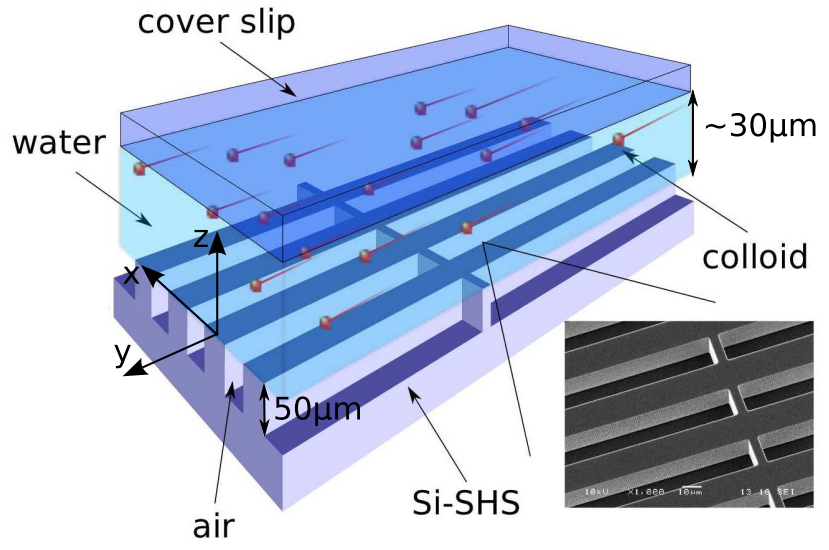

FIG. 1. Three-dimensional schematic view of the microchannel together with a scanning electron microscope image of the Si-SHS (inset). The ultrapure water and the fluorescent colloids flow in the direction parallel to the microgrooves, wherein air pockets are trapped. 
and liquid-air interfaces, separately. By correlating the flow with the actual meniscus profile, we eventually determine what boundary condition best describes the liquid-air interface behaviour. To best of our knowledge, such a strict test of the local boundary conditions at the liquid-air interfaces for micro-structured SHS has never been performed so far.

\section{METHODS AND MATERIALS}

In this section, we provide a brief description of the experimental set-up and procedures, the microfluidic device, and the velocity and interface profile measurement techniques we used for characterizing the slippage at the superhydrophobic surface. A detailed presentation of those methods is reported elsewhere. ${ }^{32}$

\section{A. Experimental set-up}

A Nikon Eclipse TE 2000-U inverted microscope, equipped with a water immersion $\times 60$ (NA 1.2) objective, is used to capture fluorescence images of the flow inside the microfluidic chip fed by a flow control system (Fluigent MFCS-Flex). A DPSS laser (Cni MLL532, $400 \mathrm{~mW}$ at $532 \mathrm{~nm}$ ) excites red fluorescent $0.3 \mu \mathrm{m}$ diameter polystyrene microspheres, dispersed in a ultrapure Milli-Q water flow ( $0.02 \%$ solid concentration). The fluorescent particles act as passive tracers for tracking both the velocity and the liquid-air and liquid-solid interface profiles. A CCD camera (Allied Vision Technologies) records the light emitted by the tracers at a frame rate of $200 \mathrm{~Hz}$. An acousto-opticmodulator (AA Optoelectronics MT80) periodically deflects the laser beam so that the camera sensor is exposed to the beam light for $10 \mu$ s only.

\section{B. Microfluidic chip}

We used a silicon superhydrophobic surface (Si-SHS), patterned with longitudinal microgrooves, $16 \mu \mathrm{m}$ in width and $50 \mu \mathrm{m}$ in depth, evenly spaced by $16 \mu \mathrm{m}$ (see Fig. 1). To prevent in case of a Wenzel transition the cavities being flooded along the whole channel length, the grooves are sectioned in smaller grooves $1 \mathrm{~mm}$ in length, separated each other by transverse $1 \mu \mathrm{m}$ thick walls, as highlighted in the inset of Fig. 1. The Si-SHS is first oxidized by oxygen plasma treatment and then silanized via standard vapor deposition of $1 \mathrm{H}, 1 \mathrm{H}, 2 \mathrm{H}, 2 \mathrm{H}$-Perfluorooctyltrichlorosilane; $97 \%$ (ABCR) to promote a stable Cassie state. The channel is finally assembled by clamping together the Si-SHS with a microscope cover slip covered with a Polydimethylsiloxane (PDMS) film about $30 \mu \mathrm{m}$ in thickness. A $2 \mathrm{~mm} \times 40 \mathrm{~mm}$ area was previously engraved on the PDMS film. The resulting channel is then $40 \mathrm{~mm}$ long, $2 \mathrm{~mm}$ wide, and $30 \mu \mathrm{m}$ deep. ${ }^{33}$ Because of the high aspect-ratio of the cross-section, the confinement effects on the fluid flow are negligible.

\section{Velocity measurements}

Using $\mu$-PIV cross-correlation analysis of sets of images recorded at different focal planes, ${ }^{17}$ we determined the velocity profile throughout the channel depth. The number $N$ of images per plane is chosen so that at the minimum detectable speed (about $30 \mu \mathrm{m} / \mathrm{s}$ ) the Brownian motion component of tracer velocity is negligible (less than 1\%) with respect to the average component. As a consequence of that ${ }^{32}$ we recorded $N=4000$ frames at focal planes nearby the silicon surface and $N=3200$ anywhere else.

A reference coordinate system as the one shown in Fig. 1 is adopted. The $z$ coordinate axis is parallel to the optical axis and positively oriented from the surface to the cover slip. Its origin is at the liquid-solid interface. The $y$ and $x$ axes are parallel and transverse to the flow direction, respectively. The axial step of the objective position is $0.3 \mu \mathrm{m}$ in the bulk and $0.1 \mu \mathrm{m}$ close to the plane $z=0$. Processing algorithms are implemented via custom Matlab codes.

\section{Interface detection}

We recently introduced a novel technique ${ }^{32}$ to measure the liquid-air and liquid-solid interface relative positions and shapes with a resolution of tens of nanometers. Such a measurement is 
performed by post-processing the same fluorescent microscopy images used for the $\mu$-PIV velocity analysis. Consequently, the velocity and interface profiles can be simultaneously determined with a single channel scan. The method is based on the detection of the excluded volume, namely, the region next to the interface which is not accessible to the moving colloids. This volume is determined by splitting the fluorescent images in overlapping rectangular interrogation windows and, then, measuring for each window the intensity of the fluorescent light emitted by the flowing tracers as a function of axial position. Nearby the interfaces, the intensity versus axial position plot can be fitted to a shifted error function. The inflection point of the best-fit function can be reasonably assumed as the center of those colloids which flowed closest to the interfaces, namely, the boundary of the excluded volume. By assembling data from all interrogation windows, a 3D reconstruction of the excluded volume boundary can be performed in the whole field of view of the microscope objective. That boundary provides the relative position and the shape of both liquid-air and liquid-solid interfaces. By considering the thickness of the depletion layer, the absolute position of both interfaces can be retrieved with the diameter of the passive tracers being a good estimate for the accuracy of such a measurement.

We have also introduced ${ }^{32}$ an additional liquid-solid interface detection algorithm, which is in good agreement with the previous one. Such a method is capable of measuring the absolute axial position of a solid wall with nanometer accuracy, provided the wall reflects the light emitted by the tracers. This is the case for a silicon flat surface which, acting as mirror, lets us track both the real and virtual (reflected) images of the tracers. The resulting velocity profiles are then symmetric with respect to the position where flow velocity vanishes to zero. The solid wall is then detected by locating the axes of symmetry of those profiles. A fine localization of the solid walls is necessary for high quality characterization of the slippage. For such a task, we adopt the reflection-based interface detection scheme since it can locate the absolute position of the liquid-solid interfaces more accurately than the excluded-volume based one.

\section{E. Cleaning and safety procedures}

Standard microfluidic cleaning procedures were adopted for manufacturing the chip and preparing the solution. Microfluidic chip fabrication and oxygen plasma treatment were performed in ISO class 8 cleanroom (NanoLyon). Silane vapor deposition was done inside a desiccator placed under a chemical fume hood whereas the chip was assembled on a laboratory worktop. The solution of Milli$\mathrm{Q}$ water and colloids was prepared and stored using clean plastic disposable vials, plastic syringes, and needles. To prevent the accumulation of fluorescent colloids on the Si-SHS, the silicon surface was cleaned via acetone ultrasonic bath and rinsed with DI water before reusing it. Microfluidic tubings and fittings were frequently cleaned with a mixture of DI water and ethanol first and then washed with DI water only. For cleaning and safety reasons, gloves were worn at all the time during sample preparation and experiments.

\section{RESULTS AND DISCUSSION}

Slippage occurs at liquid-gas and liquid-solid interfaces whenever the tangential component of the liquid velocity at the interface appears to be different from the one of the other phase (either solid or gas). That velocity gap between the two phases is called slip velocity. A well known quantity which has been used to characterize the slippage is the slip length $b$ defined as the ratio between the slip velocity and the shear rate at the interface. ${ }^{6}$ When the slip length is experimentally estimated by averaging an appropriate measurement over a length scale much larger than the molecular scale, we rather refer to it as effective slip length. For structured surfaces consisting of a periodic array of liquid-air and liquid-solid interfaces, if the measurement is averaged over one array pitch, we refer to it as global effective slip length. Conversely, if the slip length is measured just on a pitch fraction, like the liquid-air or the liquid-solid interface only, we denote it as local effective slip length. In Secs. III A-III B, we characterize the slippage of the examined Si-SHS by determining both the global and the local effective slip lengths. 

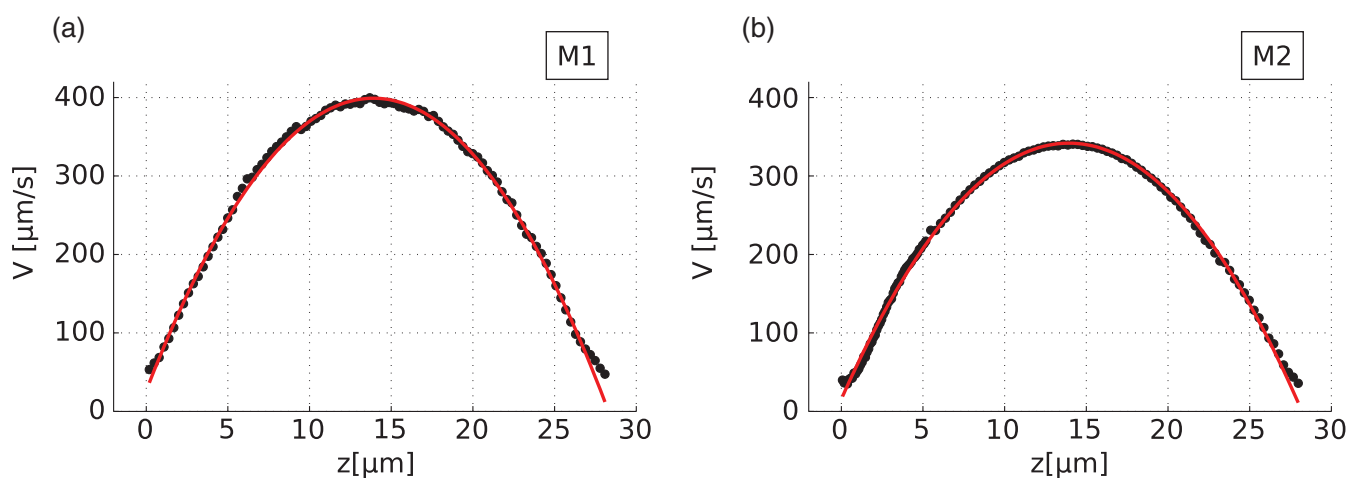

FIG. 2. Streamwise velocity profiles (solid circles) averaged over the roughness pitch for measurement M1 (left panel) and measurement M2 (right panel), together with the best-fit functions (solid lines).

\section{A. Global effective slip length}

We first assess the overall performance of our device by measuring the global effective slip length at the Si-SHS. Fluorescent images are acquired with a scan along the channel depth from $z=$ $-6 \mu \mathrm{m}$ to $z=30 \mu \mathrm{m}$. For the sake of simplicity, we recall that the origin of the $z$ axis is fixed at the liquid-solid interface of the Si-SHS. The streamwise velocity profile is averaged over the microscope field of view in the flow (y) direction and over a surface pitch, consisting of one liquid-solid and one liquid-air area, in the transverse (x) direction. The resulting profile is fitted to that one $\bar{V}(z)$ predicted by the one-dimensional (1D) model of a pressure driven flow with a sticky wall at $z=H$ (i.e., the cover slip) and a partially slippery wall at $z=0$ (i.e., the Si-SHS) with global effective slip length $b_{g l}$. The parameter $H$ stands for the channel depth. The analytical expression for $\bar{V}(z)$ reads

$$
\bar{V}(z)=4 V_{p} \frac{\left(b_{g l}+H\right)(H-z)\left[b_{g l}(z+H)+z H\right]}{H^{2}\left(2 b_{g l}+H\right)^{2}},
$$

where $V_{p}$ is the peak velocity. The fit parameters are $b_{g l}, H$, and $V_{p}$. It is worth noting that the channel depth parameter $H$ has to be fitted because we could not apply the reflection-based liquidsolid detection technique at the cover slip wall $\mathrm{z}=H$. Indeed the reflection coefficient of glass is too low to accurately track the virtual images of the colloids flowing next to the glass wall. Additionally, due to the elasticity of PDMS, the channel depth might change between experiments as it depends on the clamping force which keeps together the PDMS-coated cover slip and the Si-SHS. The measurements were performed in two different spots of the device. We refers to those measurements with the labels M1 and M2. Between M1 and M2, the microchannel was disassembled, cleaned, and reassembled. Fig. 2 shows the experimental profiles $\bar{V}(z)$ and the corresponding best-fit curves. The best-fit parameters are reported in Table I together with the fit uncertainties. It is worth recalling that in our system we do not control the geometry of the liquid-air interface and thus the meniscus can freely adapt its shape and position according to the local experimental conditions. As a consequence of that, there is no reason for the menisci to share the same shapes and positions between measurements M1 and M2.

TABLE I. Best-fit parameters and corresponding uncertainties for the 1D model, see Eq. (1), for measurements M1 and M2: peak velocity $V_{p}$, channel depth $H$, global effective slip length $b_{g l}$.

\begin{tabular}{lrr}
\hline & M1 & M2 \\
\hline$V_{p}$ & $399.3 \pm 0.5 \mu \mathrm{m} / \mathrm{s}$ & $341.4 \pm 0.5 \mu \mathrm{m} / \mathrm{s}$ \\
$H$ & $28.31 \pm 0.04 \mu \mathrm{m}$ & $28.24 \pm 0.04 \mu \mathrm{m}$ \\
$b_{g l}$ & $0.49 \pm 0.05 \mu \mathrm{m}$ & $0.33 \pm 0.04 \mu \mathrm{m}$ \\
\hline \hline
\end{tabular}


To better assess the effectiveness of the friction reduction at the Si-SHS, we compare the experimental results to the theoretical model of a surface with a periodic pattern of no-slip and no-shear stripes parallel to the flow. ${ }^{34}$ The predicted value for the global effective slip length is

$$
b_{t h}=-\frac{L}{\pi} \log \left[\cos \left(\frac{\pi}{2}\left(1-\Phi_{s}\right)\right)\right],
$$

where $L$ is the pattern pitch and $\Phi_{s}$ the solid fraction, the latter being defined as the ratio between the no-slip stripe width and the pattern pitch. For the examined Si-SHS whose solid fraction is $\Phi_{s}=0.5$ and surface pitch $L=32 \mu \mathrm{m}$, the expected global effective slip length is $b_{t h}=3.53 \mu \mathrm{m}$, namely, ten times larger than what we actually measured. Moreover, we remark that the Si-SHS sample shows highly inhomogeneous slippage as the global effective slip length varies up to $40 \%$ between M1 and M2.

\section{B. Local effective slip length}

In order to clarify this apparent contradiction between experimental and theoretical results, a local investigation of the surface friction at the liquid-air interfaces is required. Indeed the meniscus curvature at the surface cavity depends on the pressure difference between the air and liquid phases as well as the surface tension according to the Laplace law. These elements together with the meniscus position, dictated by the complex dynamics of the contact line, can be significantly affected by often uncontrolled parameters (e.g., surface defects, impurities) and thus highly varies along different regions of the Si-SHS samples. That could in principle justify the measured inhomogeneity of surface slippage as well as the strong increase of the surface friction. Nonetheless, a reduced mobility on a flat liquid-air interface could also produce the same effects. In order to discriminate between those two scenarios, we rely on our excluded-volume based interface detection technique to measure the local interfacial friction at both the liquid-solid and liquid-air interfaces. We first determine the interface profiles and then we correlate them to the corresponding flows.

Fig. 3 shows the transverse profiles of the boundaries of the excluded volumes for both M1 and M2. As reported elsewhere ${ }^{32}$ the absolute position of the measured boundaries is accurate within a tracer diameter (namely, $0.3 \mu \mathrm{m}$ ), whose size is also shown in figure for comparison. The distance between the actual interfaces and the excluded volume boundary depends on the thickness of the tracer depletion layer. At the solid surface, the depletion layer is manly due to the impenetrability of
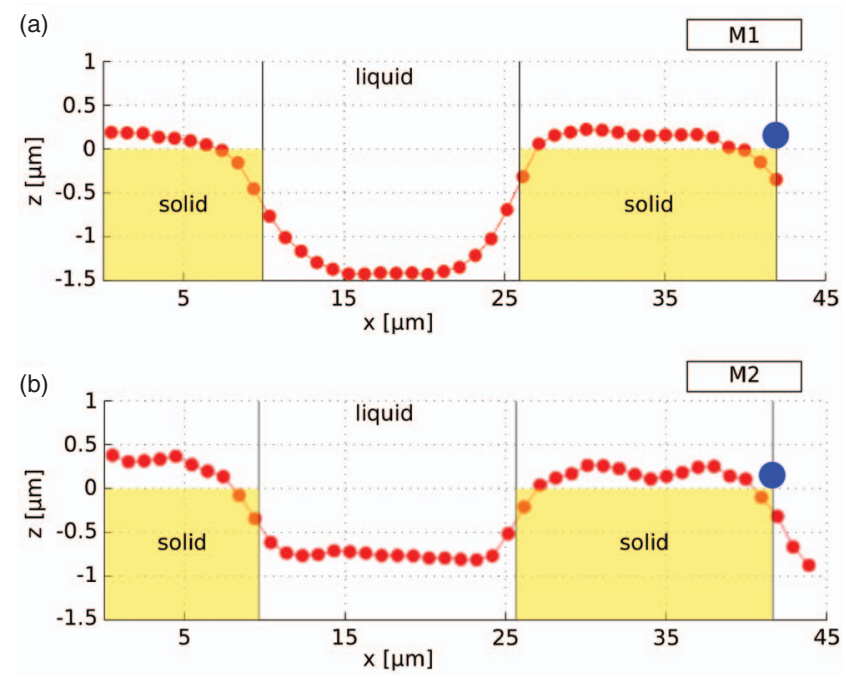

FIG. 3. Excluded volume boundary profiles along the transverse direction $x$ for M1 (top panel) and M2 (bottom panel). The passive tracer size along the axial direction $z$ is represented by the largest solid circles. 
the moving colloids with either the surface or possible tracers stuck to it. Thus the depletion layer thickness may vary approximately between 0.5 to 1.5 particle diameter (namely, between $0.15 \mu \mathrm{m}$ to $0.45 \mu \mathrm{m}$ ). Other phenomena which might affect the depletion layer, as the hydrodynamic Saffman lift or electrostatic interactions, can be neglected in our experiments. ${ }^{32}$ At the liquid-air interface, the depletion layer thickness depends on the tracer wettability. Since polystyrene is a hydrophobic material, particles tend to stuck at the liquid-air interface ${ }^{35}$ with a contact angle of about $90^{\circ}$. As a consequence of that, the depletion layer vanishes and the measured excluded-volume boundary well approximates the meniscus profile. Fig. 3 shows how both menisci are almost flat and penetrate with different depths inside the cavities. When the meniscus is modelled as a no-shear interface, partial flooding of the surface cavities reduces the surface effective slippage and higher filling levels correspond to smaller effective slip length. ${ }^{19,20}$ Our experiments show opposite behaviour with higher filling level (that is M1) corresponding to higher slippage. That is already an indirect evidence that perfect-slip condition may not apply to the examined liquid-air interfaces.

To probe what is the actual boundary condition needed to effectively model the flow past the measured liquid-air interface, we first average the streamwise velocity field along the flow direction $y$. The resulting experimental discrete function $V(x, z)$ is fitted to the two-dimensional (2D) model $V_{t h}(x, z)$ which is solution of the Poisson equation:

$$
\nabla^{2} V_{t h}(x, z)=\nabla p / \mu
$$

with the following boundary conditions:

$$
\left\{\begin{array}{l}
V_{t h}(-L / 2, z)=V_{t h}(L / 2, z) \\
\left.\frac{\partial V_{t h}}{\partial x}\right|_{x= \pm L / 2}=0 \\
V_{t h}(x, z)=b_{s} \frac{\partial V_{t h}}{\partial z} \quad \text { for } \quad(x, z) \in \text { liquid-solid interface } \\
V_{t h}(x, z)=b_{a} \frac{\partial V_{t h}}{\partial z} \quad \text { for } \quad(x, z) \in \text { liquid-air interface } \\
V_{t h}(x, H)=0
\end{array}\right.
$$

where $p$ is the liquid pressure and $\mu$ the fluid dynamic viscosity. The domain where Eq. (3) is solved for is shown in Fig. 4 together with the boundary conditions. In such a model, the meniscus is shaped according to the liquid-air interface profile measurements reported in Fig. 3, whereas the liquid-solid interfaces, located at $z=0$, are assumed to be flat. Since we cannot find an analytical closed-form for $V_{t h}(x, z)$, we used Comsol Multiphysics ${ }^{\mathrm{TM}}$ to perform the fit between the experimental 2D profile $V(x, z)$ and the numerical solution of the 2D theoretical model $V_{t h}(x, z)$. The fit parameters are the ratio $\alpha=\nabla p / \mu$ between the pressure gradient and the dynamic viscosity, the channel depth $H$ and the local effective slip lengths $b_{s}$ and $b_{a}$. The latter parameter is a direct measurement of the interfacial friction at the actual liquid-air interface. The experimental and best-fit velocity profiles are shown in Fig. 5. The solid and empty circles represent the experimental profiles at the middle point of the liquid-solid and liquid-air interfaces, respectively. Similarly, the solid and the dashed

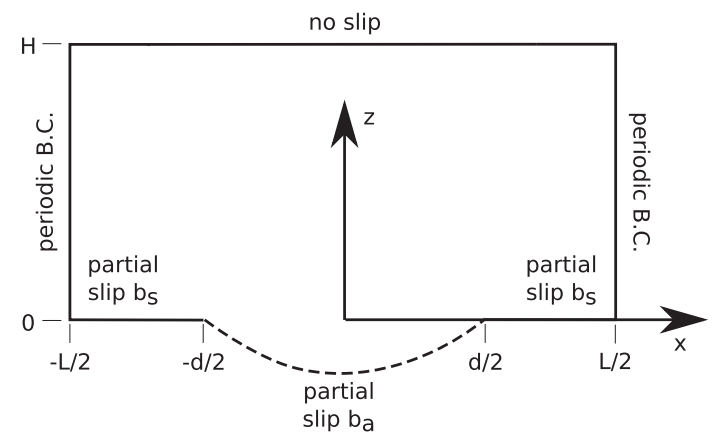

FIG. 4. 2D domain and boundary conditions where the best-fit function $V_{t h}(x, y)$ is solved for. The dashed line represents the meniscus transverse profile measured through the excluded volume based method (cf. Fig. 3). The parameter $d=L / 2$ stands for the liquid-air interface width. 

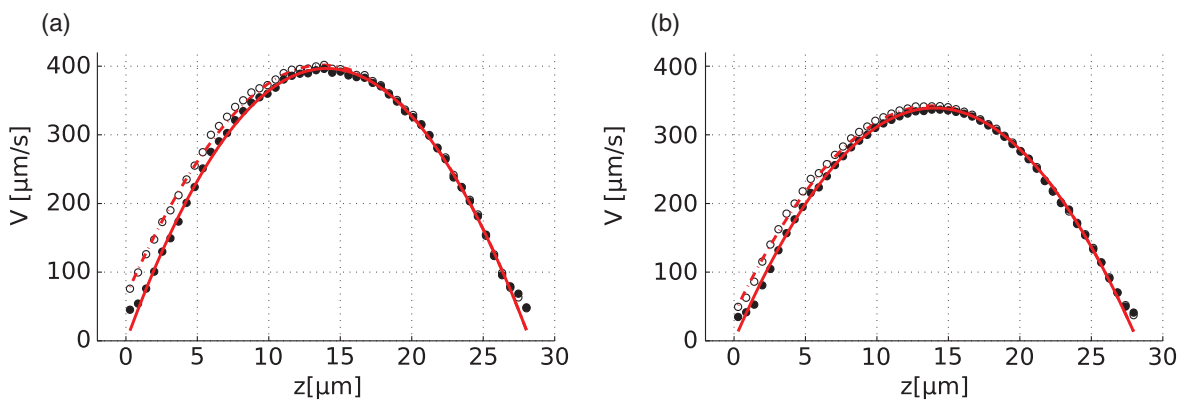

FIG. 5. Streamwise velocity profiles at the middle point of the liquid-air (empty circles) and liquid-solid (solid circles) interfaces for M1 (left panel) and M2 (right panel). The dashed and the solid lines are the corresponding best-fit profiles.

TABLE II. Best-fit parameters for the 2D model, see Eq. (3), for M1 and M2: pressure gradient and dynamic viscosity ratio $\alpha$, channel depth $H$, local effective slip lengths $b_{s}$ and $b_{a}$.

\begin{tabular}{lcc}
\hline \hline & M1 & M2 \\
\hline$\alpha$ & $3.86 \pm 0.01(\mu \mathrm{m} \cdot \mathrm{s})^{-1}$ & $3.33 \pm 0.01(\mu \mathrm{m} \cdot \mathrm{s})^{-1}$ \\
$H$ & $28.31 \pm 0.05 \mu \mathrm{m}$ & $28.24 \pm 0.05 \mu \mathrm{m}$ \\
$b_{s}$ & $-0.02 \pm 0.15 \mu \mathrm{m}$ & $0.00 \pm 0.15 \mu \mathrm{m}$ \\
$b_{a}$ & $-0.10 \pm 0.15 \mu \mathrm{m}$ & $0.09 \pm 0.15 \mu \mathrm{m}$ \\
\hline \hline
\end{tabular}

TABLE III. Interfacial shear stresses at the middle point of the liquid-solid and liquid-air interfaces for M1 and M2, calculated according to Eq. (5).

\begin{tabular}{ccc}
\hline \hline & M1 & M2 \\
\hline$\tau_{s}$ & $60 \pm 2 \mathrm{mPa}$ & $48 \pm 2 \mathrm{mPa}$ \\
$\tau_{a}$ & $50 \pm 5 \mathrm{mPa}$ & $45 \pm 5 \mathrm{mPa}$ \\
\hline \hline
\end{tabular}

lines are given by the best-fit function $V_{t h}(x, z)$ evaluated at the middle points of the liquid-solid and liquid-air interfaces, respectively. The best-fit parameters are shown in Table II. A good estimate for the accuracy of the local effective slip length measurements is given by the passive tracer diameter. We can use the best-fit function $V_{t h}(x, z)$ to calculate the interfacial shear stresses at the middle point of both the liquid-solid and liquid-air interfaces as follows:

$$
\tau=\mu \nabla V_{t h} \cdot \mathbf{n},
$$

where $\mathbf{n}$ is the unit vector normal to the interface. The results are shown in Table III, where $\tau_{s}$ and $\tau_{a}$ refer to the liquid-solid and liquid-air interfaces, respectively.

For both M1 and M2, non-zero interfacial shear stresses appear at the menisci, which turn out to be, within the accuracy of the measurements, closer to no-slip interfaces rather than no-shear ones.

\section{Discussion}

Performing simultaneous interface and velocity profile measurements with our technique reveals that the assumption of a no-shear boundary condition at the liquid-air interface is not valid for the examined SHS. That is the reason why the predicted global effective slippage of Eq. (2) is much higher than the measured values. If instead we consider a model where the stress-free boundary condition is replaced with a low partial-slip (namely, $b_{a} \ll L$ ), the corresponding global effective slip length can be estimated through the following heuristic formula: ${ }^{36}$

$$
b_{t h}=\left(1-\Phi_{s}\right) b_{a} .
$$


According to this model, for $\Phi_{s}=0.5$ the expected global slip length is $b_{a} / 2$ whereas we measured values of $b_{g l}$ larger than $b_{a}$ (cf. Tables I and II). In order to explain the observed behaviour, we must take into account the actual profile of the menisci. Fig. 3 shows that the menisci are essentially flat. Consequently, in our experiments any detrimental effect induced by the meniscus curvature ${ }^{19,23}$ on the global slippage is negligible. On the other hand, the menisci are not co-planar with the liquidsolid interfaces as it is assumed in the model, but they partially penetrate the surface cavities. Since the liquid-air interfaces are almost immobilised (i.e., $b_{a} \simeq 0$ ), the partial flooding of the cavities is responsible for an apparent positive global effective slippage, namely, $b_{g l}>0$. Had the meniscus been directed towards the flow, we would have measured a negative global effective slip length. Additionally, the fact that the position of the menisci can vary along the surface sample accounts for the observation of an apparent inhomogeneity of the surface slippage. The difference between global effective slippages in M1 and M2 is about $40 \%$ and so is the difference between the corresponding penetration depths of the menisci.

The possible causes behind the onset of an interfacial friction at the liquid-air interface for the examined sample are several. The presence of contaminant particles within the flow is one of those. Indeed, surface-active particles or molecules can significantly affect the hydrodynamic behaviour of the interface they are adsorbed to. A sound example is given by the simple experiment of a rising air bubble in a protein solution. ${ }^{37}$ Even at a low surface coverage, the bubble motion induced a non-uniform protein concentration at the interface which resulted in a surface tension gradient. Such a gradient generated the so-called Marangoni stresses which balanced the viscous stresses of the external flow. The Marangoni effect was also observed by Manor et al. ${ }^{26}$ who used an atomic force microscope to squeeze an air bubble against a mica wall across a surfactant solution film. Interestingly, the bubble interface showed partial slippage even in the absence of added surfactant. The authors explained such a behaviour by assuming the presence of some surface-active impurity, which contaminated the liquid-air interface. A recent experimental study on nanobubble-like object generation $^{38}$ reported the unexpected presence of PDMS contaminant in sterile disposable needles, which made clear how difficult is to control the presence of impurity within a sample even when working with standard microfluidic cleaning procedures.

As regards our system, the liquid-air interface is confined in a $1 \mathrm{~mm} \times 16 \mu \mathrm{m}$ rectangular region, consisting of two adjacent grooves and two consecutive transverse $1 \mu \mathrm{m}$ thick walls (see Fig. 1). Consequently, contaminant particles could be adsorbed onto the liquid-air interface and pushed by the liquid flow against the downstream transverse wall. That would result in a particle concentration gradient and, hence, Marangoni stresses which oppose the water flow nearby the meniscus, causing higher friction at the interface. ${ }^{37}$ Possible agents acting as surface contaminants could be either PDMS molecules or unknown surfactant molecules used by the supplier in the preparation of the fluorescent polystyrene colloids or even the colloids themselves. In that respect, it is worth noting that experiments involving the use of SHS are usually performed following standard microfluidic cleaning procedures. As detailed in Sec. II, we followed those procedures and we did not implement any other specialized cleaning protocol to control the level of contamination of the liquid-air interfaces. Alternative to the surface contamination scenario, we remind that Marangoni stresses could also raise from $\mu$-PIV laser-induced thermal effects. A detailed investigation about the actual causes behind the measured interfacial friction at the meniscus is beyond the scope of this research.

\section{CONCLUSIONS}

In the present paper, we applied our novel velocity and interface detection technique to thoroughly characterize the slippage behaviour of a silicon micro-grooved superhydrophobic surface. We first investigated the global slippage capability by averaging the velocity profiles along a periodic array consisting of one liquid-air and one liquid-solid interface. The surface performances were much lower than expected when modelling the surface as a periodic pattern of co-planar no-shear and no-slip parallel stripes. In addition, the surface showed an inhomogeneous behaviour when slippage was probed in different spots of the samples. To clarify the discrepancy with the theory, we evaluated the interfacial friction at the liquid-solid and liquid-air interfaces by measuring the 
meniscus profiles and relating them to the flow nearby. In doing so, we were able to probe the actual boundary condition at the deformed meniscus and we found out that the liquid-air interface was not behaving like a no-shear boundary, as it is usually assumed in the studies of SHS. Unexpectedly, the global slippage of the examined SI-SHS was a mere consequence of almost no-slip menisci protruding within the surface cavities. Due to the impossibility to control the meniscus geometry, the global slippage varied along the sample according to the local shape and position of the menisci.

We reported for the first time the violation under specific circumstances of the widely common assumption of stress-free boundary at the liquid-air interfaces in micro-structured SHS. We showed that even in presence of a stable Cassie state, the friction reduction capabilities of SHS can be seriously compromised when interfacial stresses appear at the liquid-air interfaces. We proved that those potential detrimental effects in terms of low slippage can be as worse as those deriving from a deformed meniscus protruding into the flow. ${ }^{19,23}$ The actual causes behind the onset of those stresses are still unclear and further investigations are required. Controlled sample contamination via added surfactant could help to understand the possible role played by surface-active molecules within the flow. On the other hand, the intensity of laser-induced thermal effects at the liquid-air interface has to be assessed as well. To conclude, our study demonstrated that when working with standard microfluidic cleaning procedures the presence of non-deformed (i.e., flat) liquid-air interfaces in the cavities of a SHS can no longer be considered a sufficient condition to guarantee significant surface friction reduction.

\section{ACKNOWLEDGMENTS}

We are pleased to thank L. Bocquet for interesting discussion and C. Ybert for both interesting discussions and help with the development of the experimental set-up. We also thank the Università Italo Francese and the French embassy in Italy for their financial support and the Lyon Institute of Nanotechnology (INL) for the use of their cleanroom NanoLyon. This work was partly supported by the French RENATECH network.

${ }^{1}$ J. Rothstein, “Slip on superhydrophobic surfaces,” Annu. Rev. Fluid Mech. 42, 89-109 (2010).

${ }^{2}$ C. Cottin-Bizonne, J.-L. Barrat, L. Bocquet, and E. Charlaix, "Low-friction flows of liquid at nanopatterned interfaces," Nat. Mater. 2, 237-240 (2003).

${ }^{3}$ A. Peters, C. Pirat, M. Sbragaglia, B. Borkent, M. Wessling, D. Lohse, and R. Lammertink, "Cassie-Baxter to wenzel state wetting transition: Scaling of the front velocity," Eur. Phys. J. E 29, 391-397 (2009).

${ }^{4}$ A. Giacomello, S. Meloni, M. Chinappi, and C. M. Casciola, "Cassie-Baxter and wenzel states on a nanostructured surface: Phase diagram, metastabilities, and transition mechanism by atomistic free energy calculations," Langmuir $\mathbf{2 8}$, 10764-10772 (2012).

${ }^{5}$ A. Giacomello, M. Chinappi, S. Meloni, and C. M. Casciola, "Metastable wetting on superhydrophobic surfaces: Continuum and atomistic views of the Cassie-Baxter-Wenzel transition," Phys. Rev. Lett. 109, 226102 (2012).

${ }^{6}$ E. Lauga, M. Brenner, and H. Stone, "Microfluidics: The no-slip boundary condition," Springer Handbook of Experimental Fluid Mechanics (Springer, New York, 2007), pp. 1219-1240.

${ }^{7}$ P. Joseph, C. Cottin-Bizonne, J. Benoit, C. Ybert, C. Journet, P. Tabeling, and L. Bocquet, "Slippage of water past superhydrophobic carbon nanotube forests in microchannels," Phys. Rev. Lett. 97, 156104 (2006).

${ }^{8}$ C. Lee, C. Choi, and C. Kim, "Structured surfaces for a giant liquid slip," Phys. Rev. Lett. 101, 064501 (2008).

${ }^{9}$ S. Richardson, “On the no-slip boundary condition,” J. Fluid Mech. 59, 707-719 (1973).

${ }^{10} \mathrm{~K}$. Jansons, "Determination of the macroscopic (partial) slip boundary condition for a viscous flow over a randomly rough surface with a perfect slip microscopic boundary condition,” Phys. Fluids 31, 15 (1988).

${ }^{11}$ M. Sbragaglia and A. Prosperetti, "A note on the effective slip properties for microchannel flows with ultrahydrophobic surfaces," Phys. Fluids 19, 043603 (2007).

${ }^{12}$ A. Davis and E. Lauga, "Geometric transition in friction for flow over a bubble mattress," Phys. Fluids 21, 011701 (2009).

${ }^{13}$ D. Crowdy, "Slip length for longitudinal shear flow over a dilute periodic mattress of protruding bubbles," Phys. Fluids 22, 121703 (2010).

${ }^{14}$ C. Ng and C. Wang, "Stokes shear flow over a grating: Implications for superhydrophobic slip," Phys. Fluids 21, 013602 (2009).

${ }^{15}$ L. Wang, C. Teo, and B. Khoo, "Effects of interface deformation on flow through microtubes containing superhydrophobic surfaces with longitudinal ribs and grooves," Microfluid. Nanofluid. 16, 225-236 (2014).

${ }^{16}$ J. Ou, B. Perot, and J. Rothstein, "Laminar drag reduction in microchannels using ultrahydrophobic surfaces," Phys. Fluids 16, 4635 (2004).

${ }^{17}$ P. Tsai, A. Peters, C. Pirat, M. Wessling, R. Lammertink, and D. Lohse, "Quantifying effective slip length over micropatterned hydrophobic surfaces," Phys. Fluids 21, 112002 (2009). 
${ }^{18}$ T. Kim and C. Hidrovo, "Pressure and partial wetting effects on superhydrophobic friction reduction in microchannel flow," Phys. Fluids 24, 112003-112003 (2012).

${ }^{19}$ A. Steinberger, C. Cottin-Bizonne, P. Kleimann, and E. Charlaix, "High friction on a bubble mattress," Nat. Mater. 6, 665-668 (2007)

${ }^{20} \mathrm{C}$. Teo and B. Khoo, "Flow past superhydrophobic surfaces containing longitudinal grooves: Effects of interface curvature," Microfluid. Nanofluid. 9, 499-511 (2010).

${ }^{21}$ D. Gentili, G. Bolognesi, A. Giacomello, M. Chinappi, and C. Casciola, "Pressure effects on water slippage over silanecoated rough surfaces: Pillars and holes," Microfluid. Nanofluid. 16, 1009-1018 (2014).

${ }^{22}$ J. Hyväluoma, C. Kunert, and J. Harting, "Simulations of slip flow on nanobubble-laden surfaces," J. Phys.: Condens. Matter 23, 184106 (2011).

${ }^{23}$ E. Karatay, A. S. Haase, C. W. Visser, C. Sun, D. Lohse, P. A. Tsai, and R. G. Lammertink, "Control of slippage with tunable bubble mattresses," PNAS 110, 8422-8426 (2013).

${ }^{24}$ V. Lazouskaya, Y. Jin, and D. Or, "Interfacial interactions and colloid retention under steady flows in a capillary channel," J. Colloid Interface Sci. 303, 171-184 (2006).

${ }^{25}$ L. Parkinson, R. Sedev, D. Fornasiero, and J. Ralston, "The terminal rise velocity of 10-100 $\mu \mathrm{m}$ diameter bubbles in water," J. Colloid Interface Sci. 322, 168-172 (2008).

${ }^{26}$ O. Manor, I. U. Vakarelski, G. W. Stevens, F. Grieser, R. R. Dagastine, and D. Y. Chan, "Dynamic forces between bubbles and surfaces and hydrodynamic boundary conditions," Langmuir 24, 11533-11543 (2008).

${ }^{27}$ O. Manor, I. U. Vakarelski, X. Tang, S. J. OShea, G. W. Stevens, F. Grieser, R. R. Dagastine, and D. Y. Chan, "Hydrodynamic boundary conditions and dynamic forces between bubbles and surfaces," Phys. Rev. Lett. 101, 024501 (2008).

${ }^{28}$ R. Manica, L. Parkinson, J. Ralston, and D. Y. Chan, "Interpreting the dynamic interaction between a very small rising bubble and a hydrophilic titania surface," J. Phys. Chem. C 114, 1942-1946 (2010).

${ }^{29} \mathrm{~W}$. Zheng, L. Wang, D. Or, V. Lazouskaya, and Y. Jin, "The role of mixed boundaries on flow in open capillary channels with curved air-water interfaces," Langmuir 28, 12753-12761 (2012).

${ }^{30}$ D. Yang, M. Krasowska, C. Priest, M. N. Popescu, and J. Ralston, "Dynamics of capillary-driven flow in open microchannels," J. Phys. Chem. C 115, 18761-18769 (2011).

${ }^{31}$ D. Byun, J. Kim, H. Ko, and H. Park, "Direct measurement of slip flows in superhydrophobic microchannels with transverse grooves," Phys. Fluids 20, 113601 (2008).

${ }^{32}$ G. Bolognesi, C. Cottin-Bizonne, E. Guene, J. Teisseire, and C. Pirat, "A novel technique for simultaneous velocity and interface profile measurements on micro-structured surfaces," Soft Matter 9, 2239-2244 (2013).

${ }^{33}$ G. Bolognesi, Optical Studies of Micron-Scale Flows (Lap Lambert Academic Publishing, Saarbrücken, Germany, 2012).

${ }^{34}$ J. Philip, "Flows satisfying mixed no-slip and no-shear conditions," ZAMP 23, 353-372 (1972).

${ }^{35} \mathrm{~V}$. N. Paunov, "Novel method for determining the three-phase contact angle of colloid particles adsorbed at air-water and oil-water interfaces," Langmuir 19, 7970-7976 (2003).

${ }^{36}$ C. Ybert, C. Barentin, C. Cottin-Bizonne, P. Joseph, and L. Bocquet, "Achieving large slip with superhydrophobic surfaces: Scaling laws for generic geometries," Phys. Fluids 19, 123601 (2007).

${ }^{37}$ C. Ybert and J. Di Meglio, “Ascending air bubbles in protein solutions," Eur. Phys. J. B 4, 313-319 (1998).

${ }^{38}$ R. P. Berkelaar, E. Dietrich, G. A. Kip, E. S. Kooij, H. J. Zandvliet, and D. Lohse, "Exposing nanobubble-like objects to a degassed environment," Soft Matter 10, 4947-4955 (2014). 